\title{
Entropy-Driven Triple Point Wetting in Hard-Rod Mixtures
}

\author{
Kostya Shundyak and René van Roij \\ Institute for Theoretical Physics, Utrecht University, Leuvenlaan 4, 3584 CE Utrecht, The Netherlands
}

(Received 28 June 2001; published 1 May 2002)

\begin{abstract}
We theoretically study binary mixtures of thin and thick hard rods with diameter ratio more extreme than 1:4. The bulk phase diagram of these systems exhibits a triple point, where an isotropic $(I)$ phase coexists with two nematic phases $\left(N_{1}\right.$ and $\left.N_{2}\right)$ of different composition. Using density functional theory, we predict that the $I-N_{2}$ interface is completely wet by $N_{1}$ upon approach of the the $I-N_{1}-N_{2}$ triple point. This entropic triple point wetting should be experimentally observable in colloidal suspensions of rodlike particles.
\end{abstract}

DOI: $10.1103 /$ PhysRevLett.88.205501

Sterically stabilized colloids are rigid mesoscopic particles which, when suspended in a molecular solvent, interact with each other via pairwise hard-core repulsions only [1]. As a result a suspension of hard-core colloids is athermal, i.e., its thermodynamics and structure are solely determined by entropy (free volume). Despite the lack of any cohesive energy, these systems exhibit a wealth of ordering phenomena. Classical examples of entropy-driven ordering are the freezing of a hard-sphere suspension [2] and the liquid crystalline (nematic) ordering of a hard-rod suspension [3,4] upon sufficient compression. By now it is also well known that entropy is not only capable of driving disorder-to-order transitions but also demixing of mixtures. The depletion effect, whereby two large hard-core particles attract each other effectively due to the presence of a "sea" of smaller ones, is perhaps the best known entropic demixing mechanism; it can, e.g., drive a gasliquid transition in colloid-polymer mixtures [5]. In mixtures of hard rods, the object of study in this Letter, another entropic demixing mechanism is at work: the orientation entropy can drive an immiscibility gap in the nematic phase if the two rod species are sufficiently dissimilar [6,7]. The bulk phase diagram of such mixtures not only features isotropic-nematic coexistence, but also nematic-nematic coexistence and an isotropic-nematicnematic triple point (see Fig. 1 for an example). In analogy with simple liquids and metals, where bulk critical and triple points are known to give rise to rich wetting and layering phenomena [8,9] and to surface melting [10], we expect a rich interface phenomenology in such colloidal rod fluids. An important difference is, however, that the driving mechanisms are entropic in hard-core systems, as opposed to energetic in simple liquids and metals. It is not at all obvious how the absence of cohesive energy affects the structure of such entropic interfaces. In this Letter we study, for the first time, the thermodynamics and the structure of the free interfaces between the coexisting bulk phases of binary mixtures of colloidal hard rods with widely different diameters. Upon approach of the bulk triple point we find a complete wetting phenomenon, i.e., a thick film intruding between two coexisting bulk phases. This can be seen as an entropic analog of surface
PACS numbers: 61.30.Hn, 05.70.Np, 68.03.Cd, 68.08.Bc

melting in simple metals, where a liquid film develops in the crystal-vapor interface upon approach of the triple (melting) point [10].

We consider a binary mixture of hard spherocylinders of length $L$ and diameters $D_{\sigma} \ll L$ at chemical potentials $\mu_{\sigma}$, where $\sigma=1,2$ labels the thin and thick species, respectively. The thermodynamics and the structure of this fluid are studied by means of the grand potential functional $\Omega\left[\rho_{1}, \rho_{2}\right]$ of the one-particle distribution functions $\rho_{\sigma}(q)$, where $q=(\mathbf{r}, \hat{\boldsymbol{\omega}})$ is short for the center-of-mass coordinate $\mathbf{r}$ and the orientation $\hat{\boldsymbol{\omega}}$ of the long axis of the rod. The functional is such that (i) it is minimized, for given $\left(\mu_{1}, \mu_{2}\right)$, by the equilibrium one-particle distributions, and (ii) the minimal value of the functional is the equilibrium grand potential [11]. Here we use the second virial functional

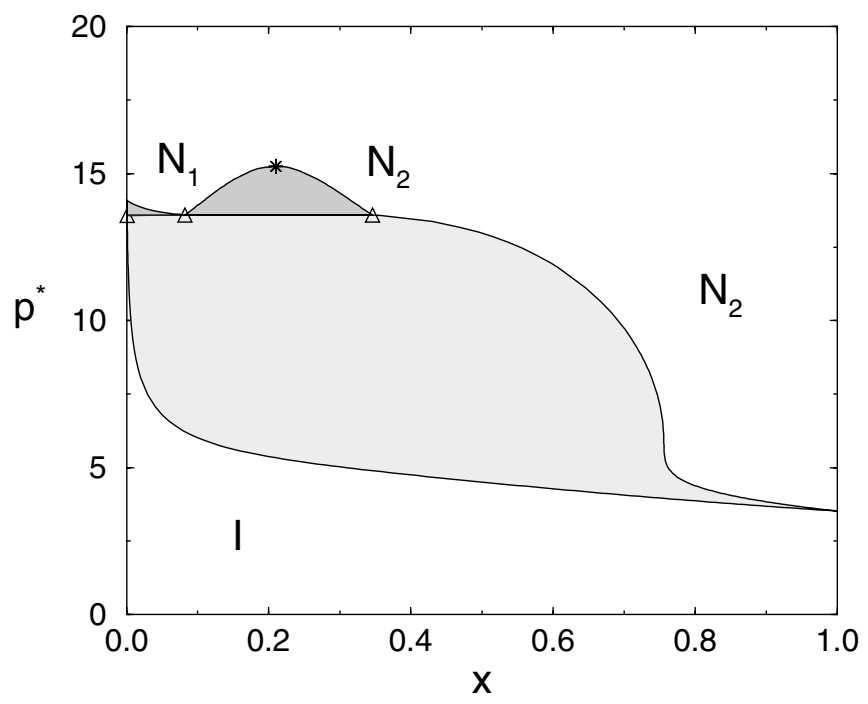

FIG. 1. Bulk phase diagram of a binary thin-thick mixture of hard rods (diameter ratio $D_{2} / D_{1}=4$, equal length $L \gg$ $D_{2}$ ), in the pressure-composition representation, with $p^{*}$ the dimensionless pressure and $x$ the mole fraction of the thicker rods. We distinguish the low-pressure isotropic phase $(I)$, highpressure nematic phases $\left(N_{1}\right.$ and $\left.N_{2}\right)$, triple coexistence $(\Delta)$, and an $N_{1}-N_{2}$ critical point $(*)$. The grey regions, enclosed by the binodals, denote the two-phase regime, and the tie lines that connect coexisting phases are horizontal. 


$$
\begin{aligned}
\Omega\left[\rho_{1}, \rho_{2}\right]= & \sum_{\sigma=1}^{2} \int d q \rho_{\sigma}(q)\left(\log \left[\rho_{\sigma}(q) L^{2} D_{\sigma}\right]-1-\mu_{\sigma}\right) \\
& -\frac{1}{2} \sum_{\sigma=1}^{2} \sum_{\sigma^{\prime}=1}^{2} \int d q d q^{\prime} \\
& \times f_{\sigma \sigma^{\prime}}\left(q, q^{\prime}\right) \rho_{\sigma}(q) \rho_{\sigma^{\prime}}\left(q^{\prime}\right)
\end{aligned}
$$

where $d q=d \mathbf{r} d \hat{\boldsymbol{\omega}}$ and $f_{\sigma \sigma^{\prime}}=-1(0)$ is the Mayer function of a (non)overlapping $\sigma \sigma^{\prime}$ pair [1,3]. We use units where $k_{B} T=1$, where $T$ is the temperature and $k_{B}$ is the Boltzmann constant. The minimum condition $\delta \Omega / \delta \rho_{\sigma}(q)=0$ yields the coupled nonlinear integral equations,

$$
\begin{aligned}
\mu_{\sigma}= & \log \left[\rho_{\sigma}(q) L^{2} D_{\sigma}\right] \\
& -\sum_{\sigma^{\prime}=1}^{2} \int d q^{\prime} f_{\sigma \sigma^{\prime}}\left(q, q^{\prime}\right) \rho_{\sigma^{\prime}}\left(q^{\prime}\right),
\end{aligned}
$$

to be solved for the equilibrium distributions $\rho_{\sigma}(q)$ at fixed $\mu_{\sigma}$. A (formal) insertion of the solutions to Eq. (2) into the functional $\Omega$ leads to the minimum value $\Omega_{0}$ given by

$$
\begin{aligned}
\Omega_{0}= & \sum_{\sigma=1}^{2} \int d q \rho_{\sigma}(q) \\
& \times\left(-1+\frac{1}{2} \log \left[\rho_{\sigma}(q) L^{2} D_{\sigma}\right]-\frac{1}{2} \mu_{\sigma}\right),
\end{aligned}
$$

which is the equilibrium grand potential of the system for given $\mu_{1}$ and $\mu_{2}$, i.e., for a given composition. For a bulk system of volume $V$ we have $\Omega_{0}=-p V$, with $p\left(\mu_{1}, \mu_{2}\right)$ the pressure. The tension $\gamma\left(\mu_{1}^{c}, \mu_{2}^{c}\right)$ due to a planar interface of area $A$ between coexisting phases with chemical potentials $\mu_{\sigma}^{c}$ follows from $\Omega_{0}=-p V+\gamma A$, where $p\left(\mu_{1}^{c}, \mu_{2}^{c}\right)$ is the coexistence pressure. We present our results in terms of the dimensionless combinations $\rho_{\sigma}^{*}(q)=\rho_{\sigma}(q) L^{2} D_{1}$ for the profiles, $n_{\sigma}^{*}(\mathbf{r})=\int d \hat{\omega} \rho_{\sigma}^{*}(q)$ for the (local) total number density of species $\sigma, p^{*}=p L^{2} D_{1} / k_{B} T$ for the pressure, and $\gamma^{*}=\gamma L D_{1} / k_{B} T$ for the tension. In the "needle" limit $D_{\sigma} / L \rightarrow 0$, which we implicitly assume from now on, the only structural length scale is $L$.

The functional of Eq. (1) is a low-density approximation, since cubic and higher order terms in $\rho_{\sigma}$ are being ignored. By considering the homogeneous, $\mathbf{r}$-independent distributions, $\rho_{\sigma}(q)=\rho_{\sigma}(\hat{\omega})$, one checks that the functional reduces to the Onsager theory for hard-rod mixtures in the isotropic and nematic bulk phases. It is well known that the only homogeneous distributions that satisfy Eq. (2) at sufficiently low $\mu_{\sigma}$ are isotropic, $\rho_{\sigma}(\hat{\omega})=n_{\sigma} /(4 \pi)$, with $n_{\sigma}$ the bulk number density of species $\sigma[1,3,12,13]$. At sufficiently high $\mu_{\sigma}$, uniaxially symmetric solutions $\rho_{\sigma}(\hat{\omega})=\rho_{\sigma}(\theta)$ exist, with $\theta=\arccos (\hat{\omega} \cdot \hat{n})$ being the polar angle of $\hat{\omega}$ with respect to the (arbitrary) nematic director $\hat{n}$. These solutions can easily be calculated numerically, e.g., on a grid of $N_{\theta}$ equidistant $\theta \in[0, \pi / 2]$. The thermodynamics follows from the evaluation of $\Omega_{0}$ from Eq. (3). Using $N_{\theta}=30$ we calculated the bulk phase diagrams of the thick-thin mixtures for several diameter ratios $D_{2} / D_{1} \equiv d$. In Fig. 1 we show the bulk phase diagram for $d=4$ in the pressure-composition representation, where the composition variable $x=n_{2} /\left(n_{1}+n_{2}\right)$ denotes the fraction of thick rods. The striking features of this phase diagram, in which the grey areas denote the two-phase regions, include the strong fractionation at $I-N_{2}$ coexistence, an $N_{1}-N_{2}$ immiscibility gap enclosed by a critical (consolute) point $(*)$, and $I-N_{1}-N_{2}$ triple coexistence $(\Delta)$ at the triple pressure $p_{t}^{*} \simeq 13.58$. Consistent with Ref. [7], where bulk phase diagrams of thin-thick mixtures are presented for a number of $d$ 's, we find that for increasing $d>4$ the triple point $I$ and $N_{1}$ phases approach the pure-thin bulk coexistence (i.e., $x \rightarrow 0$ ), whereas the composition of the triple point $N_{2}$ phase shifts to a purethick phase $(x \rightarrow 1)$. In addition, the critical pressure increases dramatically with $d$ up to $d=d_{c}=4.29$, where it diverges. For $d>d_{c}$ there is no $N_{1}-N_{2}$ critical point [6,7].

We now turn to the thermodynamics and the structure of the (free) interfaces between the coexisting phases. Assuming these interfaces to be planar, with normal $\hat{z}$, we can describe these by the inhomogeneous one-particle distributions $\rho_{\sigma}(z, \hat{\omega})$. Here $z=\hat{z} \cdot \mathbf{r}$ is the spatial coordinate normal to the interface. The interfacial distributions are solutions of the Euler-Lagrange equations (2) at the coexistence chemical potentials $\mu_{\sigma}=\mu_{\sigma}^{c}$, with $\rho_{\sigma}(z \rightarrow \pm \infty, \hat{\omega})=\rho_{\sigma}^{( \pm)}(\hat{\omega})$ being the two coexisting bulk distributions (labeled by $(+)$ and $(-)$ here for brevity). The kernels $f_{\sigma \sigma^{\prime}}\left(q, q^{\prime}\right)$ in the Euler-Lagrange equations (2) can be reduced, in the planar geometry here, to the in-plane integrated kernels $K_{\sigma \sigma^{\prime}}\left(z-z^{\prime}, \hat{\omega}, \hat{\omega}^{\prime}\right)=$ $-\int d x^{\prime} d y^{\prime} f_{\sigma \sigma^{\prime}}\left(\mathbf{r}, \hat{\omega} ; \mathbf{r}^{\prime}, \hat{\omega}^{\prime}\right)$, for which explicit analytic expressions are given in Refs. [14,15]. The problem of the free planar interface can then, in principle, be solved straightforwardly by, e.g., iterating Eq. (2) on a $(z, \theta, \varphi)$ grid, where $\theta=\arccos (\hat{\omega} \cdot \hat{n})$ is the polar angle of $\hat{\omega}$ with respect to the nematic bulk director $\hat{n}$, and $\varphi$ the azimuthal angle. It turns out, however, that the $\varphi$ dependence, i.e., the biaxiality, is very weak in the isotropic-nematic interface of the one-component hard-rod fluid $[15,16]$, even in the "most biaxial" (and thermodynamically most stable) geometry $\hat{n} \perp \hat{z}$. The same is expected for the mixture of interest here. We can therefore reduce the computational costs by projecting out the $\varphi$ dependence of $\rho_{\sigma}$. This projection can also be seen as an expansion in the biaxiality, truncated at lowest order [15]. The resulting uniaxial distributions $\rho_{\sigma}(z, \theta)$, with symmetry axis $\hat{n}$ for all $z$, are solutions of the azimuthally smeared EulerLagrange equation,

$$
\begin{aligned}
\mu_{\sigma}^{c}= & \log \left[\rho_{\sigma}(z, \theta) L^{2} D_{\sigma}\right]+2 \pi \sum_{\sigma^{\prime}} \int d z^{\prime} \int d \theta^{\prime} \sin \theta^{\prime} \\
& \times \mathcal{K}_{\sigma \sigma^{\prime}}\left(z-z^{\prime}, \theta, \theta^{\prime}\right) \rho_{\sigma^{\prime}}\left(z^{\prime}, \theta^{\prime}\right)
\end{aligned}
$$

where $\quad \mathcal{K}_{\sigma \sigma^{\prime}}\left(z, \theta, \theta^{\prime}\right)=(2 \pi)^{-2} \int_{0}^{2 \pi} d \varphi \int_{0}^{2 \pi} d \varphi^{\prime} \times$ $K_{\sigma \sigma^{\prime}}\left(z, \hat{\omega}, \hat{\omega}^{\prime}\right)$ is the doubly azimuthally averaged kernel 


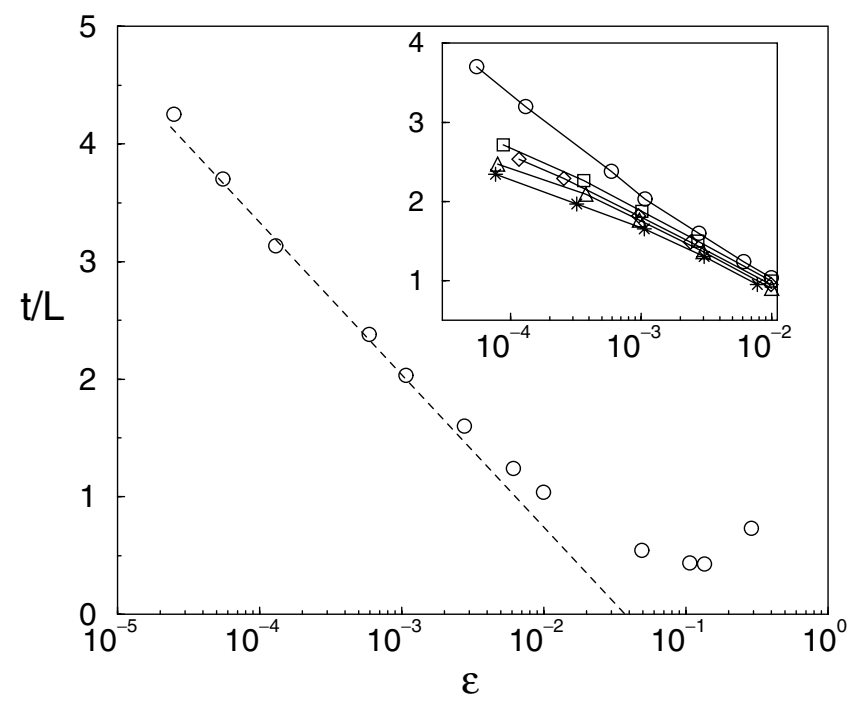

FIG. 2. Thickness $t$ (see text) of the $I-N_{2}$ interface for diameter ratio $d=4$ as a function of the undersaturation $\epsilon=1-p / p_{t}$ from the triple point pressure $p_{t}$. From the slope of the logarithmic growth of $t$ for $\epsilon \rightarrow 0$ the bulk correlation length $\xi=0.49 L$ of the wetting $N_{1}$ phase is deduced. The inset shows the logarithmic growth of $t / L$ for diameter ratios $d=4(\bigcirc)$, $4.2(\square), 4.5(\diamond), 4.7(\Delta)$ and $5(*)$.

[15]. By iteration of Eq. (4) we calculated $\rho_{\sigma}(z, \theta)$ for a number of state points $\left\{\mu_{\sigma}^{c}\right\}$ on the $I-N_{1}, I-N_{2}$, and $N_{1}-N_{2}$ binodals. In all cases we used an equidistant spatial grid of $N_{z}=200,400$ points $z_{i} \in[-5 L, 5 L]$ or $[-10 L, 10 L]$ with spacing $z_{i+1}-z_{i}=L / 20$, and an equidistant angular grid of $N_{\theta}=30$ points $\theta_{j} \in[0, \pi / 2]$ as for the bulk calculations. Note that $\mathcal{K}_{\sigma \sigma^{\prime}}\left(z-z^{\prime}, \theta, \theta^{\prime}\right)$ need to be determined, on a similar grid, only once numerically. From the equilibrium distributions $\rho_{\sigma}(z, \theta)$ we calculate the local densities $n_{\sigma}(z)=4 \pi \int_{0}^{\pi / 2} d \theta \sin \theta \rho_{\sigma}(z, \theta)$ for the two species $\sigma=1,2$, and the standard local nematic order parameters $S_{\sigma}(z)=4 \pi \int_{0}^{\pi / 2} d \theta \sin \theta P_{2}(\cos \theta) \rho_{\sigma}(z, \theta) / n_{\sigma}(z)$, with $P_{2}(x)=\left(3 x^{2}-1\right) / 2$ the second Legendre polynomial. The interface thickness $t$ is taken as $t=\left|z_{+}-z_{-}\right|$, where the positions $z_{ \pm}$are defined by $n_{2}^{\prime \prime \prime}\left(z_{ \pm}\right)=0$, i.e., at the extrema of $n_{2}^{\prime \prime}(z)$, where a prime indicates a derivative with respect to $z$. This rather arbitrary measure for the interface thickness, which is such that $t / L=0.697$ for the one-component isotropic-nematic interface, is chosen because it provides a measure for the thickness of both monotonic and nonmonotonic profiles, with and without a thick film in between the asymptotic bulk phases at $z \rightarrow \pm \infty$.

The $I-N_{1}$ interfaces, which only exist in a small pressure regime $p \geq p_{t}$, closely resemble the isotropic-nematic interface of the pure hard-rod fluid $[15,16]$, i.e., the profiles $S_{\sigma}(z)$ and the total density $n_{1}(z)+n_{2}(z)$ change monotonically from the bulk values in $I$ to those in $N_{1}$. The thickness of this interface is very similar to that of the pure system, and for $d=4$ its tension takes the value $\gamma_{I-N_{1}}^{*}=(0.2092 \pm 0.0002)$ at $p=p_{t}$. The $N_{1}-N_{2}$ inter- face for $d=4$ does not contain surprises either: its tension vanishes upon the approach of the critical point, and for $p \downarrow p_{t}$ the profiles $S_{\sigma}(z)$ and $n_{\sigma}(z)$ are monotonic, $t / L=0.592$ is microscopically thin, and the tension at $p=p_{t}$ is given by $\gamma_{N_{1}-N_{1}}^{*}=0.0194 \pm 0.0002$, i.e., an order of magnitude smaller than $\gamma_{I-N_{1}}^{*}$. The microscopic interface thickness for $p \geq p_{t}$ is to be contrasted with that of the $I-N_{2}$ interface at $p<p_{t}$, which we find to diverge, for all $d \geq 4$, when $p \uparrow p_{t}$. This can be seen in Fig. 2, where $t / L$ is plotted as a function of $\varepsilon=1-p / p_{t}$, i.e., $\varepsilon$ is a measure for the "undersaturation" with respect to the triple point pressure $p_{t}$. The logarithmic divergence of $t$ with $\epsilon \rightarrow 0$ is the first signature of a complete wetting phenomenon in the $I-N_{2}$ interface.

The nature of the film can be analyzed from the density profiles $n_{\sigma}(z)$ and the order parameters $S_{\sigma}(z)$. This is illustrated in Fig. 3, where the thick-rod density profile, $n_{2}^{*}(z)$, of the $I-N_{2}$ interface is shown for $d=4$ at several values of the undersaturation $\varepsilon$. The asymptotic densities at $z \rightarrow \pm \infty$ in Fig. 3 are those of the coexisting $I$ and $N_{2}$ bulk phases (at the corresponding $\epsilon$ ). The local density of thick rods in the film is, for $\epsilon \rightarrow 0$, exactly that of the bulk triple point $N_{1}$ phase. This is indicated by the dashed line in Fig. 3. The same identification can be made for $n_{1}^{*}(z)$ and $S_{\sigma}(z)$, and on this basis we conclude that the complete wetting phenomenon under consideration is complete triple point wetting of the free $I-N_{2}$ interface by $N_{1}$.

So far, however, this conclusion is based only on the structure and thickness of the films. In order to confirm this conclusion thermodynamically we determine the ratio of surface tensions

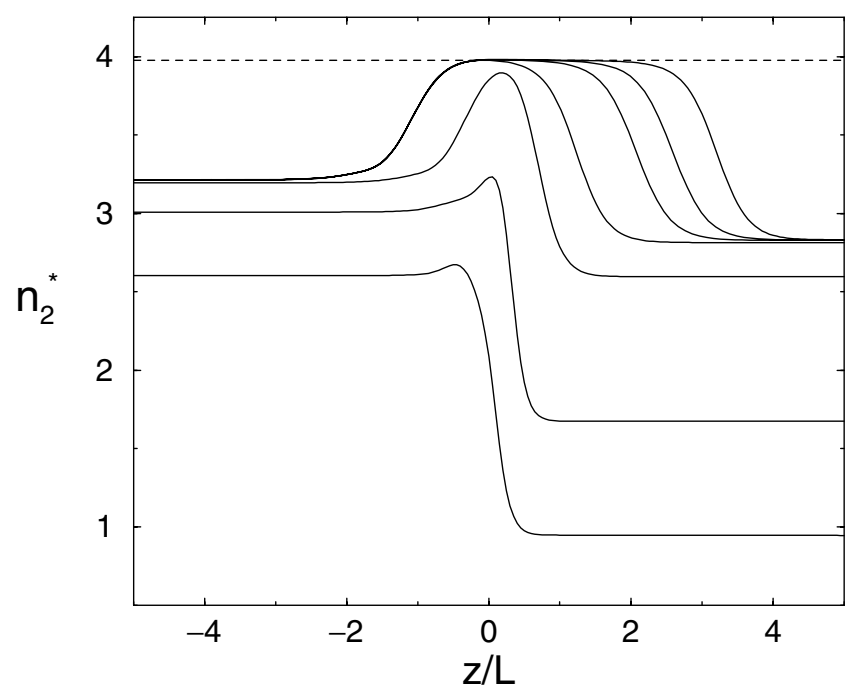

FIG. 3. Density profiles $n_{2}^{*}(z)$ of the thick rods in the $I-N_{2}$ interface for diameter ratio $d=4$ at triple point undersaturations $\varepsilon=1-p / p_{t}=0.29,0.1,0.01,5 \times 10^{-4}, 1.3 \times 10^{-4}, 5 \times$ $10^{-5}, 2.5 \times 10^{-5}$ from bottom to top. The bulk $I$ phase is at $z \rightarrow \infty$, and the bulk $N_{2}$ phase is at $z \rightarrow-\infty$. The dashed line $n_{2}^{*}=3.977$ represents the density of thick rods in the triple point $N_{1}$ phase. These profiles indicate the formation of a wetting $N_{1}$ film in the $I-N_{2}$ interface. 


$$
R(\epsilon)=\frac{\gamma_{I-N_{2}}(\epsilon)}{\lim _{p \downarrow p_{t}}\left(\gamma_{I-N_{1}}+\gamma_{N_{1}-N_{2}}\right)}
$$

plotted in Fig. 4. For all diameter ratios considered here, we see from this figure that $\lim _{\epsilon \rightarrow 0} R=1$, which implies a vanishing contact angle. This constitutes the thermodynamic proof of complete triple point wetting [9] in all thick-thin hard-rod mixtures with $d \geq 4$. In fact, we also carried out a study of long-short mixtures of hard rods of the same diameter. For length ratios more extreme than $1: 3$, these systems exhibit a bulk triple point [6] for which we find the same triple point wetting phenomenon as found in thin-thick mixtures.

The data presented here allow for a consistency check by means of a twofold calculation of the bulk correlation length $\xi$, in the direction perpendicular to the nematic director, of the triple point $N_{1}$ phase. On the one hand, one expects, for the short-range interactions of interest here, that the thickness of the $N_{1}$ film in the $I-N_{2}$ interface diverges as $t=t_{0}-\xi \ln \epsilon$ for $\epsilon \rightarrow 0$ [9]. From the slope of the small- $\epsilon$ part of Fig. 2 we find that, for $d=4, \xi / L=0.49 \pm 0.02$. This value decreases, for increasing $d$, to the limiting value $\xi / L=0.32 \pm 0.02$ for $d \geq 5$. This value is the perpendicular correlation length of the one-component coexisting nematic phase, i.e., the $N_{1}$ triple point for large $d$. On the other hand, one expects [17] that the asymptotic decay of $\rho_{\sigma}(z, \theta)$ to its $N_{1}$ bulk value $\rho_{\sigma}^{\left(N_{1}\right)}(\theta)$ is proportional to $\exp [-|z| / \xi]$, for all $\theta$ and both species $\sigma=1,2$. From the $N_{1}$ side of the profiles obtained in the $I-N_{1}$ and $N_{1}-N_{2}$ interface (at $p \downarrow p_{t}$, not shown here), we find, consistently with the above results, that $\xi / L=0.48 \pm 0.02$ for $d=4$, which changes to $\xi / L=0.32 \pm 0.02$ for $d \geq 5$. The fact that $\xi$ is of the order of $L$ implies that, together with the fact that the

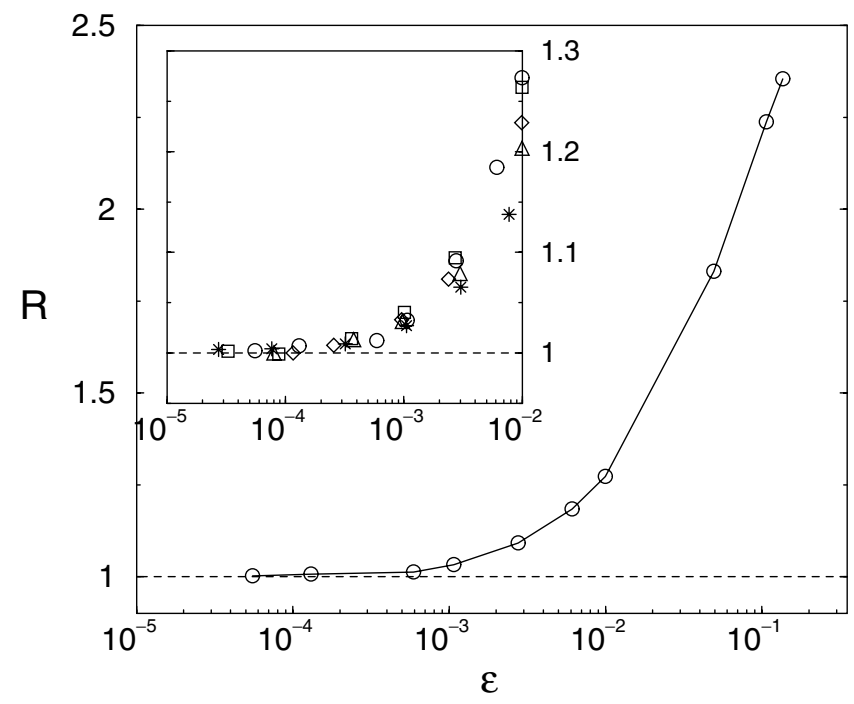

FIG. 4 Surface tension ratio $R$ [see Eq. (5)] as a function of the triple point undersaturation $\epsilon$ for diameter ratio $d=4$. The inset shows the same quantity for $d=4(\bigcirc), 4.2(\square), 4.5(\diamond)$, $4.7(\Delta)$, and $5(*)$. tension $\gamma$ is of the order of $k_{B} T / L D_{1}$, the so-called wetting parameter [9] $\omega \equiv k T /\left(4 \pi \gamma \xi^{2}\right) \sim D_{1} / L \rightarrow 0$. As a consequence the wetting $N_{1}$ film is in the stiff, mean-field limit, where thickness fluctuations are unimportant. This is an interesting addition to Onsager's classic notion that the second virial approximation is exact for thermodynamic bulk properties of fluids of hard rods in the needle limit [3].

In conclusion, on the basis of an inhomogeneous version of Onsager's second virial functional we predict that binary thin-thick mixtures of hard rods with a diameter ratio $d \geq 4$ exhibit entropic triple point wetting of the $I-N_{2}$ interface by the $N_{1}$ phase. This phenomenon should be experimentally observable in, e.g., gene-manipulated Tobacco Mosaic Virus mixtures, for which synthesis in this regime of diameter ratios has been recently achieved [18].

It is a pleasure to thank M. Dijkstra, R. Evans, and H.N.W. Lekkerkerker for stimulating discussions and careful reading of the manuscript. This work is part of the research program of the "Stichting voor Fundamenteel Onderzoek der Materie (FOM)," which is financially supported by the "Nederlandse organisatie voor Wetenschappelijk Onderzoek (NWO)."

[1] G. J. Vroege and H. N. W. Lekkerkerker, Rep. Prog. Phys. 55, 1241 (1992).

[2] W. W. Wood and J. D. Jacobson, J. Chem. Phys. 27, 1207 (1957); B. J. Alder and T. E. Wainwright, J. Chem. Phys. 27, 1208 (1957); P. N. Pusey and W. van Meegen, Nature (London) 320, 340 (1986).

[3] L. Onsager, Ann. N.Y. Acad. Sci. 51, 627 (1949).

[4] S. Fraden, G. Maret, D. L. D. Caspar, and R. B. Meyer, Phys. Rev. Lett. 63, 2068 (1985).

[5] M. Dijkstra, J. M. Brader, and R. Evans, J. Phys. Condens. Matter 11, 10079 (1999).

[6] G. J. Vroege and H. N. W. Lekkerkerker, J. Phys. Chem. 97, 3601 (1993).

[7] R. van Roij, B. Mulder and M. Dijkstra, Physica (Amsterdam) 261A, 374 (1998).

[8] J. W. Cahn, J. Chem. Phys. 66, 3667 (1977).

[9] M. Schick, in Liquids at Interfaces, edited by J. Charvolin, J. Joanny, and J. Justin (Elsevier, New York, 1990).

[10] J. W. M. Frenken and J. F. van der Veen, Phys. Rev. Lett. 54, 134 (1985).

[11] R. Evans, Adv. Phys. 28, 143 (1979).

[12] R.F. Kayser and H. J. Raveché, Phys. Rev. A 17, 2067 (1978).

[13] H. N. W. Lekkerkerker, Ph. Coulon, R. Van Der Haegen, and R. Deblieck, J. Chem. Phys. 80, 3427 (1984).

[14] A. Poniewierski and R. Holyst, Phys. Rev. A 38, 3721 (1988).

[15] K. Shundyak and R. van Roij, J. Phys. Condens. Matter 13, 4789 (2001).

[16] Z. Y. Chen and J. Noolandi, Phys. Rev. A 45, 2389 (1992); Z. Y. Chen, Phys. Rev. E 47, 3765 (1993).

[17] R. Evans and R. J. F. Leote de Carvalho, J. R. Henderson, and D. C. Hoyle, J. Chem. Phys. 100, 591 (1994).

[18] Z. Dogic and S. Fraden, Philos. Trans. R. Soc. London A 359, 997 (2001). 